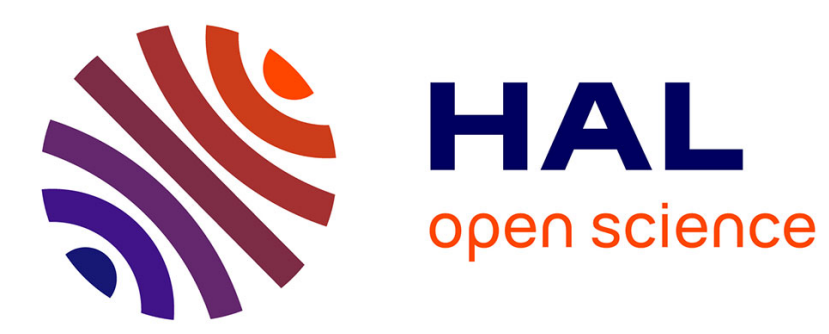

\title{
Passivity and Maximum Quality Factor Assessment in Lossy 2-port Transfer Functions
}

Matteo Oldoni, Fabien Seyfert, Steven Caicedo Mejillones, Stefano Moscato, Giuseppe Macchiarella

\section{- To cite this version:}

Matteo Oldoni, Fabien Seyfert, Steven Caicedo Mejillones, Stefano Moscato, Giuseppe Macchiarella. Passivity and Maximum Quality Factor Assessment in Lossy 2-port Transfer Functions. IEEE Transactions on Circuits and Systems II: Express Briefs, In press, pp.5. 10.1109/TCSII.2021.3089428 . hal-03261743

\section{HAL Id: hal-03261743 \\ https://hal.inria.fr/hal-03261743}

Submitted on 16 Jun 2021

HAL is a multi-disciplinary open access archive for the deposit and dissemination of scientific research documents, whether they are published or not. The documents may come from teaching and research institutions in France or abroad, or from public or private research centers.
L'archive ouverte pluridisciplinaire HAL, est destinée au dépôt et à la diffusion de documents scientifiques de niveau recherche, publiés ou non, émanant des établissements d'enseignement et de recherche français ou étrangers, des laboratoires publics ou privés. 


\title{
Passivity and Maximum Quality Factor Assessment in Lossy 2-port Transfer Functions
}

\author{
Matteo Oldoni ${ }^{1}$, Fabien Seyfert ${ }^{2}$, Steven Caicedo Mejillones ${ }^{1,3}$, Stefano Moscato ${ }^{1}$ and Giuseppe Macchiarella ${ }^{3}$
}

\begin{abstract}
Lossy transfer functions are appealing in the design of filters and electric networks, as they can be exactly implemented by physical passive components. However, lossy techniques relax most of the constraints governing the design and thus offer many degrees of freedom but with unclear effects on realizability. This work describes first an analytical method to check whether a given 2-port matrix transfer function is passive. Moreover, for comparison purposes, a technique to assess the maximum allowed predistortion is proposed, related to the highest required quality factor.
\end{abstract}

Index Terms-Lossy network, predistortion, quality factor, passivity, microwave filters.

\section{INTRODUCTION}

In the theory of electric networks, relevant research has been and currently is dedicated to lossless transfer functions. However, within the field of RF and microwave filters, losslessness is not a correct assumption as physical passive components are in fact strictly lossy.

As a matter of fact, the lossless approach remains a handy tool in everyday work [1], and often the designer chooses this robust and consolidated method by introducing losses only in the last steps and minimizing their impact by optimization.

Substantially different routes are instead the "lossy" techniques, dedicated to transfer functions exactly implementable by passive components [2]-[5] which suit disparate technological possibilities, e.g. [6]-[9]. In this area, most of the properties supporting the designer [10] (transmission zeros in paraconjugate pairs if not on the imaginary axis, guaranteed minimum McMillan degree...) do not hold any more and open up several degrees of freedom; their adjustment dramatically affects implementability, but this often becomes clear only at the end of the synthesis, thus demanding more iterations.

This paper proposes in section II an analytical technique to verify if a given $2 \times 2$ rational scattering function is passive.

After facing this issue, another need arises: how to compare the synthesizability of lossy functions? The practical answer proposed by this work is to evaluate the maximum required quality factor for synthesis. Section IV describes an analytical method relying on the solution of two bivariate polynomials.

M. Oldoni, S. Caicedo and S. Moscato are with R\&D Dept., SIAE Microelettronica; via Buonarroti 21, IT-20093, Cologno Monzese (MI), Italy.

F. Seyfert is with the Functional analysis for conception and analysis of systems (FACTAS) Team, INRIA; Sophia Antipolis cedex, BP 93, France

G. Macchiarella and S. Caicedo are with the Electronics, Information and Bioengineering Dept., Politecnico di Milano; via Ponzio 34/5, IT-20133, Milan, Italy

Manuscript received...; revised....

\section{PASSIVITY REQUIREMENTS}

A 2-port reciprocal system is described in the complex domain $s=\sigma+j \omega$ by the symmetrical scattering matrix $\boldsymbol{S}(s)$. As often the case, the involved functions are moreover rational, i.e. each is a ratio of polynomials in $s$ and share the denominator $D(s)$ which holds the $N$ poles of the system as its roots and which is assumed monic (i.e. with unitary leading coefficient) without loss of generality:

$$
\boldsymbol{S}(s)=\left[\begin{array}{ll}
S_{11}(s) & S_{21}(s) \\
S_{21}(s) & S_{22}(s)
\end{array}\right]=\frac{\left[\begin{array}{cc}
N_{11}(s) & N_{21}(s) \\
N_{21}(s) & N_{22}(s)
\end{array}\right]}{D(s)}
$$

All the involved polynomials are considered to be known, i.e. computed according to the requirements.

A common further assumption is that the network can be implemented with $N$ resonators, thus not requiring redundancy. This implies that the transfer function has minimum McMillan degree, which means that there exist a polynomial $P(s)$ :

$$
N_{11}(s) N_{22}(s)-\left(N_{21}(s)\right)^{2}=D(s) P(s) \text {. }
$$

The minimum McMillan degree property can be checked by computing the complex roots of the left-hand member and verifying that all the poles are among them. If so, the $P(s)$ polynomial can be computed as taking all the other (not of $D(s)$ ) roots of the left-hand member and with a leading coefficient equal to that of the left-hand member.

The involved polynomials are classically with real coefficients in the $s$ variable, guaranteeing that no constant reactances are needed. However, most synthesis techniques are applied in a lowpass domain where constant reactances are allowed, as they approximately translate to physical components in bandpass/bandstop domains. As a consequence, we consider complex polynomial coefficients to be likewise allowed, as they do not affect passive realizability in such cases.

For a passive reciprocal transfer function, two simultaneous conditions on the scattering matrix must be verified, [11]:

1) Each element of $\boldsymbol{S}(s)$ is analytic in $\operatorname{Re}(s)>0$, thus all the poles lie strictly in the left half-plane (Hurwitz);

2) $\boldsymbol{A}(s)=\boldsymbol{I}-\boldsymbol{S}^{*}(s) \boldsymbol{S}(s)=\boldsymbol{I}-\overline{\boldsymbol{S}}(-\bar{s}) \boldsymbol{S}(s)$ is positive semi-definite for every $s=j \omega$ where $\omega \in \mathbb{R}$, implying that the two eigenvalues of $\boldsymbol{A}(j \omega)$ are always both positive for whatever real value of $\omega$. The complex conjugate of a complex $x$ is denoted as $\bar{x}$; the ${ }^{*}$ operator is here the classical paraconjugate of complex rational functions, that is $\boldsymbol{S}^{*}(s)=(\overline{\boldsymbol{S}}(-\bar{s}))^{t}=\overline{\boldsymbol{S}}(-\bar{s})$. Practically, here the ${ }^{*}$ operation amounts to conjugate the polynomial coefficients of the matrix rational function and to change the variable $s$ in $-s$. 
Condition 1 is immediate to check, hence not studied further.

Condition 2 instead requires to evaluate $A(j \omega)$ over the whole $\omega$ axis and check the eigenvalues at each step: this represents a cumbersome task whose outcome depends on the choice of finite sampled values of $\omega$. To avoid such bruteforce attempts, the following section III develops a novel formulation which requires only three root computations.

\section{PASSIVITY ASSESSMENT}

Several approaches can be devised to verify the positive semi-definiteness of $\boldsymbol{A}(s)$ by exploiting algebraic properties and the inner structure of $\boldsymbol{S}(s)$. For any of these, first the matrix $\boldsymbol{A}(s)=\boldsymbol{I}-\boldsymbol{S}^{*}(s) \boldsymbol{S}(s)$ must be developed:

$$
\begin{aligned}
& \boldsymbol{A}(s)=\left[\begin{array}{cc}
1-\frac{N_{11} N_{11}^{*}+N_{21} N_{21}^{*}}{D D^{*}} & -\frac{N_{11}^{*} N_{21}+N_{21}^{*} N_{22}}{D D^{*}} \\
-\frac{N_{21}^{*} N_{11}+N_{22}^{*} N_{21}}{D D^{*}} & 1-\frac{N_{22} N_{22}^{*}+N_{21} N_{21}^{*}}{D D^{*}}
\end{array}\right] \\
& =\frac{\left[\begin{array}{cc}
D D^{*}-N_{11} N_{11}^{*}-N_{21} N_{21}^{*} & -N_{11}^{*} N_{21}+N_{21}^{*} N_{22} \\
-N_{21}^{*} N_{11}-N_{22}^{*} N_{21} & D D^{*}-N_{22} N_{22}^{*}-N_{21} N_{21}^{*}
\end{array}\right]}{D D^{*}}
\end{aligned}
$$

For $s=j \omega, \boldsymbol{A}(s)$ is Hermitian and its positive definiteness on the whole imaginary axis is equivalent to the system passivity, provided condition 1) is met.

\section{A. Sylvester's criterion}

For a Hermitian matrix like $\boldsymbol{A}(j \omega)$ to be positive semidefinite, the Sylvester's criterion gives a set of conditions equivalent to the unpractical inspection of eigenvalues in $\omega$ :

$$
\begin{array}{|l|l|l|}
\hline A_{1,1}(j \omega) \geq 0 & A_{2,2}(j \omega) \geq 0 & \operatorname{Det}(\boldsymbol{A}(j \omega)) \geq 0 \\
\hline
\end{array}
$$

The first two conditions are equivalent to:

$$
C_{i}(s)=D D^{*}-N_{i i} N_{i i}^{*}-N_{21} N_{21}^{*} \geq 0 \text { for } i \in\{1,2\}
$$

By moving into the Fourier domain $s=j \omega$, the expression becomes a real polynomial in $\omega$ : if any of the two conditions has a real root $\omega$ with odd multiplicity, then $\boldsymbol{A}(j \omega)$ is not always positive semi-definite and $\boldsymbol{S}$ is not a passive network.

For the third condition we make use of the formula

$$
\operatorname{Det}(\boldsymbol{I}+\boldsymbol{B})=1+\operatorname{Tr}(\boldsymbol{B})+\operatorname{Det}(\boldsymbol{B})
$$

which holds for any matrix $\boldsymbol{B}$ of size $2 \times 2$ and where Det and Tr denote respectively determinant and trace of their matrix arguments. This yields:

$$
\begin{aligned}
& \operatorname{Det}(\boldsymbol{A}(s))=1-\operatorname{Tr}\left(\boldsymbol{S}^{*} \boldsymbol{S}\right)+\operatorname{Det}\left(\boldsymbol{S}^{*} \boldsymbol{S}\right) \\
& =\frac{D D^{*}-N_{11} N_{11}^{*}-N_{22} N_{22}^{*}-2 N_{21} N_{21}^{*}+P P^{*}}{D D^{*}}
\end{aligned}
$$

where $\operatorname{Det}\left(\boldsymbol{S}^{*} \boldsymbol{S}\right)$ has been computed using (2). Therefore,

$$
C_{3}(s)=D D^{*}-N_{11} N_{11}^{*}-N_{22} N_{22}^{*}-2 N_{21} N_{21}^{*}+P P^{*} \geq 0 .
$$

Checking the passivity of a $2 \times 2$ transfer function with poles in the left-hand plane thus amounts to assess the non-negativity of three real valued polynomials, $C_{i}(s)$, on the imaginary axis.

Proposition 3.1: Let $q(\omega)$ be a real polynomial of degree $2 N$. Denote by $q_{k}, k=0 \ldots n$ the coefficient of $q(\omega)$ in $\omega^{k}$. The polynomial $q$ is non-negative on the real line if and only if $q_{0} \geq 0$ and $q_{2 N} \geq 0$ and either one of the following condition holds:

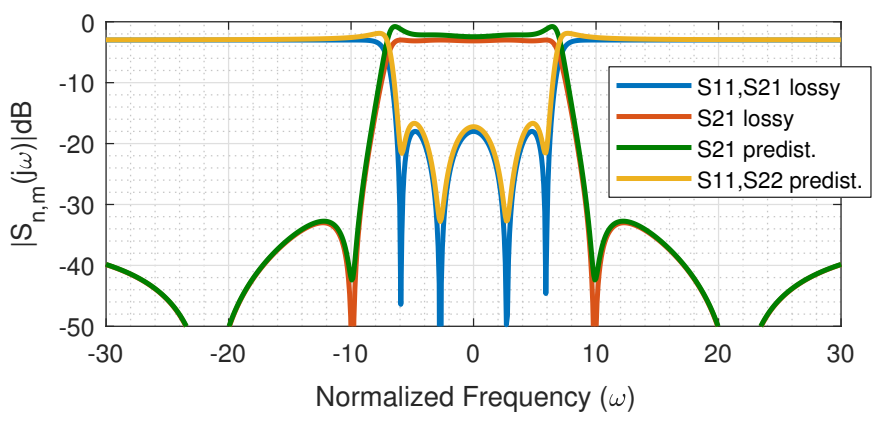

Fig. 1. Lossy scattering parameters obtained by scaling by $3 \mathrm{~dB}$ a Cauer (elliptic) 4th degree function (blue and red traces) and predistorted version with $\sigma=-0.25$ (green and orange traces). The predistorted version is the one considered for the example of section III-B

1) Any positive real root of $q$ is of even multiplicity.

2) If $\omega_{i}>0$ are the positive real roots of $q^{\prime}$, the derivative of $q$, then $q\left(\omega_{i}\right) \geq 0$ holds for all $i$.

The two criteria necessitate, when applied to the passivity testing of our system of McMillan degree $N$, the computation of roots of polynomials of degree $2 N$ and $2 N-1$.

Criterion 1) can be moreover tested using a purely algebraic procedure, that is without recurring to a numerical root solving procedure and by using Sturm sequences. To do so what is first needed is a square free decomposition of the polynomial $q$, that is the determination of the square free factors $q_{i}$ such that $q=\prod_{i=1}^{n}\left(q_{k}\right)^{i}$. Here all the $q_{k}$ are coprime and have simple roots; these factors can be obtained via greatest common divisor calculations. For example $q_{1}=q / \operatorname{gcd}\left(q, q^{\prime}\right)$ and all other factors can be obtained in a similar fashion. Once the factors $q_{k}$ have been obtained, criterion 1) amounts to test for the presence of positive zeros in the $q_{k}$ s for every odd values of $k$. This can be done by first removing, if any, the possible roots of $q_{k}$ at zero, and then computing the sign changes in the Sturm signed reminder sequences [12] at 0 and $+\infty$.

The burden of the proposed procedures is lower than sampling the $\omega$ axis and they are thus more robust and convenient.

\section{B. Numerical Example}

An example is given for a 4-th degree transfer function with negative real parts of poles and $N_{22}(s)=-N_{11}(s)$ :

Roots $\left\{N_{11}(s)\right\}=\{0.2547 \pm j 5.9220 ; 0.2547 \pm j 2.7191\}$;

Roots $\left\{N_{21}(s)\right\}=\{0.2547 \pm j 21.5363 ; 0.2547 \pm j 9.8883\}$;

Roots $\{D(s)\}=\{-0.7698 \pm j 6.8332 ;-3.8187 \pm j 3.7327\}$;

Roots $\{P(s)\}=\{1.2793 \pm j 6.8332 ; 4.3281 \pm j 3.7327\}$;

$N_{11}(s)=0.7076 s^{4}-0.7210 s^{3}+30.3223 s^{2}-15.3545 s+185.421 ;$

$N_{21}(s)=0.022387 s^{4}-0.02281 s^{3}+12.5811 s^{2}-6.4066 s+1016.1$;

$D(s)=s^{4}+9.1769 s^{3}+87.558 s^{2}+405.03 s+1348.3$

$P(s)=-0.5012 s^{4}+5.6207 s^{3}-51.6931 s^{2}+251.556 s-791.215$

The magnitude response of this scattering transfer function is shown in fig. 1 with the "predistorted" label (its meaning will be clearer in a successive example), and no non-passivity areas are evident from it. However, the evaluation of $C_{i}(j \omega)$ highlights two small regions where eq. $(4)<0$ and two larger ones where eq. $(7)<0$ : the network is in fact not passive. 


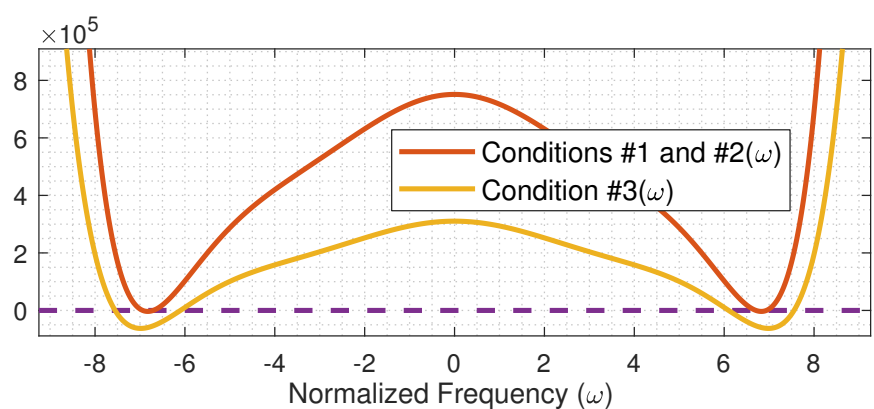

Fig. 2. The evaluation of $C_{1}(j \omega)$ and $C_{2}(j \omega)$ of eq. (4) and of $C_{3}(j \omega)$ of eq. (7) of a non-passive transfer function, observed by $C_{3}(j \omega)$ being negative at some real values of $\omega$.

To verify the proposed method, the $\omega$ roots of eqs. (4) and (7) (all with positive leading coefficients and constant term) can be respectively computed as:

$$
\begin{aligned}
& \{4 \text { cplx roots } ; \pm 6.9389 ; \pm 6.7069\} \\
& \{4 \text { cplx roots } ; \pm 7.5540 ; \pm 6.0998\}
\end{aligned}
$$

The computed real roots correspond to the sign changes in fig. 2 and, as there exist at least one real $\omega$ root (there are four for each $C_{i}$ here), the proposed method successfully detects this transfer function as not passive.

As alternative confirmation, the real roots of the derivative of $C_{3}(j \omega)$ are $\{ \pm 6.9849 ; 0\}$, and the values of $C_{3}(j \omega)$ at these points are $\left\{-6.25 \cdot 10^{4} ; 3.1 \cdot 10^{5}\right\}$, which contain negative values hence making the network not passive. Similarly, the Sturm's theorem detects 4 distinct real roots to each condition.

\section{FINDING REQUIRED Q}

After having established in a binary way whether a given transfer function is passive or not, the related problem is to quantify its passivity, for comparing different functions. The classical tool here proposed to this end is predistortion, which creates a function $\hat{\boldsymbol{S}}(s)$ by shifting the complex plane:

$$
\hat{\boldsymbol{S}}_{\sigma}(s)=\boldsymbol{S}(s+\sigma) \text {, with real } \sigma
$$

A negative predistortion $\sigma<0$ creates $\hat{\boldsymbol{S}}_{\sigma}$ with all its poles and zeros shifted toward the right of the $s$-plane. If $\boldsymbol{S}(s)$ is passive, $\hat{\boldsymbol{S}}(s)_{\sigma}$ has poles closer to the imaginary axis, hence sharper peaks in the magnitude response of fig. 1. By making $\sigma$ more and more negative, $\hat{\boldsymbol{S}}(j \omega)_{\sigma}$ will become no longer passive; the method of section III can be used for this check.

The maximum predistortion violating passivity $\left(\sigma_{0}\right)$, i.e. making the distorted function lossless at a real frequency, is computable for any transfer function and enables comparisons.

The practical usefulness of this proposed measure of passivity is that $-\sigma_{0}$ is inversely related to the maximum quality factor required by a circuit implementation. This relation, in common experience, holds generally although arbitrary lossy synthesis techniques often involve approximations, optimizations or no guarantees that components are all real; the relation is instead clearer when performing synthesis by even-odd mode decomposition [3] (requiring $S_{11}=S_{22}$ ), in which the synthesis is reduced to two subnetworks with prescribed input reflection functions: at each step, a frequency where the

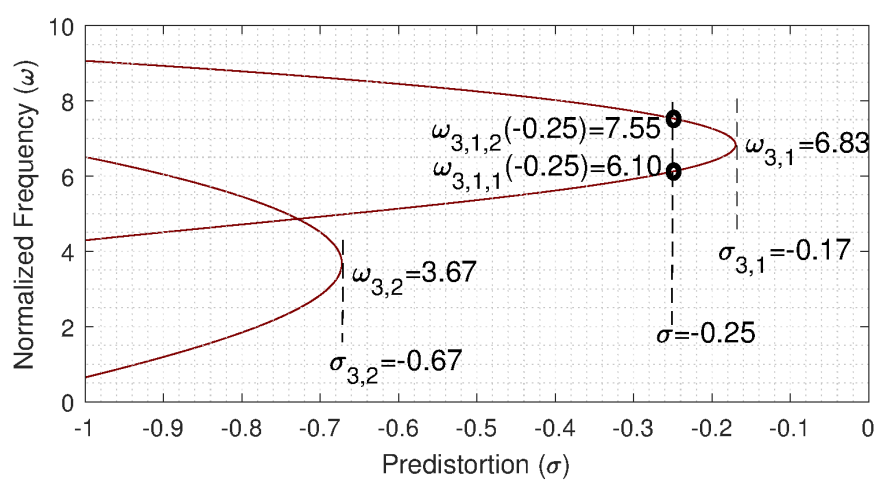

Fig. 3. Locus of points $(\sigma, \omega)$ solving $C_{3, \sigma}(j \omega)=0$ of the lossy function in fig. 1. Two curves are shown (others appear symmetrically for $\omega<0$ due to the symmetry of the example) as obtained by drawing the 0 -height contour of eq. (7) via numerical evaluation on the complex plane. Their apexes are denoted as $\sigma_{3,1}$ and $\sigma_{3,2}$. Another value of $\sigma=-0.25$, used in fig. 1 is also depicted along with the two corresponding $\omega$ intersections

network is lossless is needed (as the one obtained by applying $\left.\sigma_{0}\right)$ to then extract and remove one lossless resonator, i.e. by section extraction. Further predistortion $\sigma_{n}=\sigma_{n-1}+\delta_{n}$ can be cumulated after each step and the network is so synthesized by lossless resonators. It is then converted to implement the original non-distorted lossy response by applying the opposite $\sigma_{n}$ to all frequency-dependent components, i.e. resonators, which therefore become lossy with a quality factor $Q_{n} \propto-\frac{1}{\sigma_{n}}$, in general non-uniform across the whole network. The maximum required quality factor hence is given by the least negative value, which is $\sigma_{0}$.

The following sections outline an analytical approach to determine $\sigma_{0}$ which relies on bivariate polynomials.

\section{A. Passivity as predistortion}

The rationale for this approach is that the predistorted polynomials defining $\hat{\boldsymbol{S}}_{\sigma}(s)$, when evaluated along the imaginary axis $j \omega$, actually represent the values of the original polynomials along a shifted axis. As assessment of passivity must take place in the predistorted domain, the predistorted polynomials $\left(\hat{N}_{11}(s), \hat{N}_{21}(s), \hat{N}_{22}(s), \hat{D}(s)\right.$ and $\left.\hat{P}(s)\right)$ must first be paraconjugated and evaluated along $j \omega$. For instance:

$$
\hat{N}_{11}^{*}(j \omega)=\overline{\hat{N}_{11}}(-\overline{j \omega})=\overline{N_{11}}(-\overline{j \omega}+\sigma) .
$$

Passivity conditions built on the predistorted functions require that, for a given $\sigma, \hat{D}(s)$ be Hurwitz and neither of eqs. (4) and (7) recomputed on the $\sigma$-predistorted polynomials and denoted as $C_{i, \sigma}$ have any odd-multiplicity imaginary root $j \omega$.

Considering only one of the 3 conditions, the locus of points $\sigma+j \omega$ where $C_{i, \sigma}(j \omega)=0$ is represented by curves (0-level contours) in the complex plane, as in fig. 3. These curves will lie entirely in a half-space $\sigma<\sigma_{i, j}$, each curve $j$ with its own $\sigma_{i, j}$. Considering only one of such curves, with an excessive predistortion $\sigma<\sigma_{i, j}$ the curve will cross $\sigma$ at least twice (say at $\omega_{i, j, 1}$ or $\left.2(\sigma)\right)$ : thus one of the eigenvalues of the matrix $\boldsymbol{A}$ changes sign at $\omega_{i, j, 1,2}(\sigma)$, hence being negative in an $\omega$ range and representing a non-passive predistorted function.

For the numerical example considered, the value of $\sigma=$ -0.25 , used to predistort the response in fig. 1 , intersects the 
0 -level contour at $\omega_{3,1,1}(-0.25)=6.08$ and $\omega_{3,1,2}(-0.25)=$ 6.75. Therefore, that amount of predistortion is excessive, as it causes violation of passivity by the distorted transfer function.

When $\sigma>\sigma_{i}$ is chosen, instead, the curve does not intersect $\sigma$, and thus both eigenvalues are strictly positive, as in a passive predistorted function. When several curves are present, $\sigma>\sigma_{i}=\max _{j} \sigma_{i, j}$ guarantees that condition $i$ is fulfilled and $\sigma_{i}$ is the allowed predistortion. From this pictorial view, the determination of $\sigma_{i}$ can be turned into an algebraic problem.

This approach must be applied to all conditions $C_{i, \sigma}(j \omega)$ and the maximum $\sigma_{0}=\max \sigma_{i}$ among the three constitutes the allowed predistortion $\sigma_{0}$ of the transfer function (possibly limited by the poles remaining in the left half-plane).

\section{B. Bivariate polynomials approach}

Notably, the $C_{i, \sigma}(j \omega)$ do not coincide with either a mere predistortion applied to the polynomials eqs. (4) and (7) or their evaluation along $s=\sigma+j \omega$. The resulting expressions are in fact no longer polynomials in a single complex variable: conditions $C_{i, \sigma}(j \omega)$ are made by terms in the form $\hat{f}(s) \hat{f}^{*}(s)$ evaluated along $j \omega$ with $\hat{f}(s)$ any of the $\sigma$-predistorted polynomials. The non-paraconjugated terms $\hat{f}(s)$ evaluated in $j \omega$ are still polynomials in $s=\sigma+j \omega$ :

$$
\left.\hat{f}(s)\right|_{j \omega}=\left.f(s+\sigma)\right|_{j \omega}=\left.\sum f_{k}(s+\sigma)^{k}\right|_{j \omega}=\sum f_{k}(\sigma+j \omega)^{k}
$$

However, the paraconjugated terms $\hat{f}^{*}(s)$ when evaluated in $j \omega$ are instead polynomials in $\sigma-j \omega=\bar{s}$ :

$$
\left.\hat{f}^{*}(s)\right|_{j \omega}=\left.\overline{f(-\bar{s}+\sigma)}\right|_{j \omega}=\left.\sum \overline{f_{k}}(-s+\sigma)^{k}\right|_{j \omega}=\sum \overline{f_{k}}(\sigma-j \omega)^{k}
$$

Although the $C_{i, \sigma}$ are not polynomials in a single complex variable but actually involve both $s$ and $\bar{s}$ (from terms paraconjugated and not), each of these can be converted into real bivariate polynomials. For a general polynomial $f(s)$, a function $f(\sigma, \omega)$ can be defined on real parameters, $\sigma$ and $\omega$ :

$$
f(s)=\sum f_{k} s^{k}=f(\sigma+j \omega)=f(\sigma, \omega)=\sum g_{n, m} \sigma^{n} \omega^{m}
$$

where $f(\sigma, \omega)$ is a bivariate polynomial. The conversion from univariate complex to bivariate real is merely the binomial expansion of the variable in $f(s)$ :

$$
f(s)=\sum f_{k}(\sigma+j \omega)^{k}=\sum f_{k} \sum_{n}\left(\begin{array}{l}
k \\
n
\end{array}\right) j^{k-n} \sigma^{n} \omega^{k-n}=g(\sigma, \omega)
$$

Similarly, for its paraconjugate part (as given in eq. (9)), $f^{*}(s)$ will involve the conjugated coefficients and the oddpower terms of $\omega$ will receive a further sign change.

Therefore, once the equivalent bivariate forms for $D(s)$, $D^{*}(s), N_{11}(s), N_{11}^{*}(s), N_{21}(s), N_{21}^{*}(s), N_{22}(s), N_{22}^{*}(s)$, $P(s), P^{*}(s)$ have been computed, the required products and sums create three large bivariate polynomials $C_{i}(\sigma, \omega)$.

To determine the allowed predistortion of condition $i$, one can state that it lies within the roots of $C_{i}(\sigma, \omega)$, i.e. it is onto the curves in the complex plane as in fig. 3, which are however rather complicated to compute. More specifically, in fact, the interest is in determining the apexes of those curves and selecting the right-most one. Thus a further algebraic condition is introduced: the partial derivative of the bivariate polynomial with respect to $\omega$ in the desired solution must be 0 , which specifies that the relevant points are only those where the curves are tangent to the $\sigma$ axis.

A system of two bivariates is hence built for each $C_{i}$ :

$$
\left\{C_{i}(\sigma, \omega)=0\right\} \cap\left\{\partial C_{i}(\sigma, \omega) / \partial \omega=0\right\}, i \in\{1,2,3\} .
$$

This system has a 0 -size solution, i.e. a finite number of points, determinable by algebraic techniques or dedicated solvers.

Concerning the former, for the two large bivariate polynomials to have a common root, their resultant must be 0 . Hence values of $\sigma_{i, j}$ can be obtained by computing the real roots of the determinant of the Sylvester's matrix:

$$
R(\sigma)=\operatorname{Res}_{\omega}\left\{C_{i}(\sigma, \omega) ; \partial C_{i}(\sigma, \omega) / \partial \omega\right\}
$$

Although $R(\sigma)$ can be of high degree even for low-order functions, efficient algorithms dedicated to real roots are available. To this end, the polysol2 tool, part of the TensorLab package [13], computes the resultant and solves a generalized eigenvalue problem, whose results are then selectively discarded and the rest improved by local optimization.

Of the obtained finite solutions $\sigma_{i, j}$ (the corresponding $\omega_{i, j}$ are discarded), the one with the largest $\sigma$ must be taken as allowed predistortion for condition $i: \sigma_{i}=\max \sigma_{i, j}$.

The maximum of those is the least predistortion which makes the network lossless, hence its inverse is the largest required quality factor: the more negative the allowed predistortion is, the "more lossy" the transfer function is, the poorer resonators are needed for its synthesis.

\section{Numerical Example}

The numerical example is the lossy set of polynomials obtained by scaling by $3 \mathrm{~dB}$ a 4 th-degree elliptic response with $15 \mathrm{~dB}$ return loss in the $\pm 2 \pi$ passband and more than $30 \mathrm{~dB}$ attenuation beyond $\pm 3 \pi$ :

$$
\begin{aligned}
& N_{11}(s)=-N_{22}(s)=\left(0.9995 s^{4}+42.4423 s^{2}+259.1558\right) / \sqrt{2} \\
& N_{21}(s)=\left(0.03166 s^{4}+17.7801 s^{2}+1435.8135\right) / \sqrt{2} \\
& D(s)=s^{4}+10.1959 s^{3}+94.9604 s^{2}+451.491 s+1457.34 \\
& P(s)=-s^{4}+10.1959 s^{3}-94.9604 s^{2}+451.491 s-1457.34
\end{aligned}
$$

(side note: these polynomials, predistorted excessively with $\sigma=-0.25$, give those of the example of section III-B, which are detected to be non-passive).

Their bivariate version is computed; for instance:

$$
\begin{aligned}
D(\sigma, \omega)= & \omega^{4}-4 j \sigma \omega^{3}-10.196 j \omega^{3}-6 \sigma^{2} \omega^{2}-30.588 \sigma \omega^{2}-94.960 \omega^{2} \\
& +4.000 j \sigma^{3} \omega+30.588 j \sigma^{2} \omega+189.921 j \sigma \omega+451.491 j \omega \\
& +\sigma^{4}+10.196 \sigma^{3}+94.960 \sigma^{2}+451.491 \sigma+1457.34
\end{aligned}
$$

and the bivariate form of the $\hat{D}^{*}(s)$ is found by conjugating all coefficients of $D(s)$, then applying bivariate expansion and further changing sign of the coefficients of odd-powers of $\omega$ :

$$
\begin{aligned}
\hat{D}(\sigma, \omega)= & \omega^{4}+4 j \sigma \omega^{3}+10.196 j \omega^{3}-6 \sigma^{2} \omega^{2}-30.588 \sigma \omega^{2}-94.960 \omega^{2} \\
& -4 j \sigma^{3} \omega-30.588 j \sigma^{2} \omega-189.921 j \sigma \omega-451.491 j \omega \\
& +\sigma^{4}+10.196 \sigma^{3}+94.96 \sigma^{2}+451.491 \sigma+1457.34
\end{aligned}
$$

By multiplying the bivariate forms of non-paraconjugated and paraconjugated polynomials according to eqs. (4) and (7), the bivariate polynomials $C_{i}(\sigma, \omega)$ are finally obtained. 


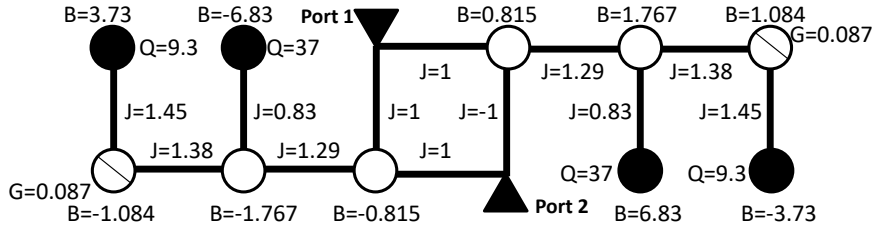

Fig. 4. Synthesized lumped-element circuit for the lossy response of fig. 1 and section IV-C. Solid lines represent admittance inverters with the prescribed admittance inversion constant $(J)$; solid circles represent unitary resonators with the prescribed quality factor $Q$. Hollow circles denote non-resonant nodes with shunt susceptance $B$. Barred circles are lossy non-resonant nodes with shunt admittance $G+j B$. Triangles denote external ports with unitary reference impedance.

The polysol2 tool yields, applied to this lossy scattering parameters set, for condition $C_{1}$ of eq. (4) and its derivative in eq. (13) (the same for $C_{2}$ ):

$$
\begin{array}{ll}
\sigma_{1,1}=-0.2520 & \text { with } \omega_{1,1}= \pm 6.82 \\
\sigma_{1,2}=-28.9658 & \text { with } \omega_{1,2}=0
\end{array}
$$

whereas, for $C_{3}$ of eq. (7) and its derivative in eq. (13):

$$
\begin{array}{ll}
\sigma_{3,1}=-0.1698 & \text { with } \omega_{3,1}= \pm 6.8305 \\
\sigma_{3,2}=-0.6723 & \text { with } \omega_{3,2}= \pm 3.6777 \\
\sigma_{3,3}=-0.7286 & \text { with } \omega_{3,3}= \pm 4.8611 \\
\sigma_{3,4}=-1.1304 & \text { with } \omega_{3,4}=0 \\
\sigma_{3,5}=-1.4572 & \text { with } \omega_{3,5}= \pm 2.3139 \\
\sigma_{3,6}=-2.8467 & \text { with } \omega_{3,6}= \pm 10.7140 \\
\sigma_{3,7}=-29.122 & \text { with } \omega_{3,7}= \pm 1.9
\end{array}
$$

The maximum values for each condition are $\sigma_{1}=\sigma_{2}=$ -0.252 and $\sigma_{3}=-0.1698$, hence the maximum allowed predistortion is $\sigma_{0}=-0.1698$, as also confirmed by fig. 3 . A synthesis of the corresponding lumped-element circuit with passband edge at $\pm 1 \mathrm{~Hz}$ is carried out by means of the evenodd decomposition technique in [3]. After removing a unitary admittance inverter on port 2 , the circuit shown in fig. 4 is obtained, whose response exactly corresponds to the "lossy" one in fig. 1. The quality factor of the involved resonators at the passband edge is found to be 37 and 9.3. As expected, the maximum quality factor involved satisfies $37=\frac{2 \pi \cdot 1 \mathrm{~Hz}}{\sigma_{0}}$.

\section{CONCLUSion}

This work addresses two closely-related issues arising when dealing with non-lossless transfer functions. One is the determination of whether a given 2-port scattering matrix is passive, whose proposed solution is based on a simple rootfinding operation over 3 independent polynomials, which is much more effective than inspecting the sampled responses; alternative root-counting algorithms are also proposed to the same purpose and a numerical example proves the approaches.

The other is the identification of the maximum allowed predistortion for passivity comparisons, which is also directly related to the required quality factor for resonators. The solution to this problem is provided as an analytic procedure with bivariate polynomials, also validated by numerical examples.

These tools can prove to be effective for the synthesis of transfer functions with practical lossy components, where many degrees of freedom with unclear effects must be handled.

\section{REFERENCES}

[1] R. J. Cameron, C. M. Kudsia, and R. R. Mansour, Microwave Filters for Communication Systems. Wiley-Interscience, 2007.

2] A. C. Guyette, I. C. Hunter, and R. D. Pollard, "The design of microwave bandpass filters using resonators with nonuniformq," IEEE Transactions on Microwave Theory and Techniques, vol. 54, no. 11, pp. 3914-3922, Nov. 2006.

[3] M. Oldoni, G. Macchiarella, G. G. Gentili, and C. Ernst, "A new approach to the synthesis of microwave lossy filters," IEEE Transactions on Microwave Theory and Techniques, vol. 58, no. 5, pp. 1222-1229, 2010.

[4] M. Meng and I. C. Hunter, "Synthesis of coupling matrix for lossy filter networks," in 2015 IEEE MTT-S International Microwave Symposium, 2015, pp. 1-3.

[5] B. S. Senior, I. C. Hunter, and J. D. Rhodes, "Synthesis of lossy filters," in Proc. 32nd European Microwave Conference, Oct. 2002, pp. 1-4.

[6] V. Miraftab and M. Yu, "Generalized lossy microwave filter coupling matrix synthesis and design using mixed technologies," IEEE Transactions on Microwave Theory and Techniques, vol. 56, no. 12, pp. 30163027, Dec. 2008.

[7] A. A. Muller, A. Moldoveanu, V. Asavei, E. Sanabria-Codesal, and J. Favennec, "Lossy coupling matrix filter synthesis based on hyperbolic reflections," in 2016 IEEE MTT-S International Microwave Symposium (IMS), 2016, pp. 1-4.

[8] A. Périgaud, A. Basti, S. Bila, N. Delhote, D. Baillargeat, and S. Verdeyme, "Design of a hybrid siw - microstrip lossy filter in ltcc technology," in 2017 IEEE MTT-S International Microwave Workshop Series on Advanced Materials and Processes for RF and THz Applications (IMWS-AMP), 2017, pp. 1-3.

[9] L.-F. Qiu, L.-S. Wu, W.-Y. Yin, and J.-F. Mao, "A flat-passband microstrip filter with nonuniform- $q$ dual-mode resonators," IEEE Microwave and Wireless Components Letters, vol. 26, no. 3, pp. 183-185, 2016.

[10] A. Fettweis, "Scattering properties of real and complex lossless 2-ports," Electronic Circuits and Systems, IEE Proceedings G, vol. 128, no. 4, pp. $147-148$, august 1981.

[11] P. Triverio, S. Grivet-Talocia, M. S. Nakhla, F. G. Canavero, and R. Achar, "Stability, causality, and passivity in electrical interconnect models," IEEE Transactions on Advanced Packaging, vol. 30, no. 4, pp. 795-808, Nov 2007.

[12] S. Basu, R. Pollack, and M. Coste-Roy, Algorithms in Real Algebraic Geometry, ser. Algorithms and Computation in Mathematics. Springer Berlin Heidelberg, 2013.

[13] N. Vervliet, O. Debals, L. Sorber, M. Van Barel, and L. De Lathauwer. (2016) Tensorlab 3.0. [Online]. Available: http://www.tensorlab.net 